\title{
Efficacy of Antibiotics and Bactericides for the Management of Xanthomonas citri (Hasse) Causing Citrus Canker
}

\author{
Jahir Basha*, C.R. Prabhu, C. Ganiger and M.C. Soniya \\ Agricultural Research Station, Pavagada, University of Agricultural Sciences, \\ GKVK, Bangalore, India \\ *Corresponding author
}

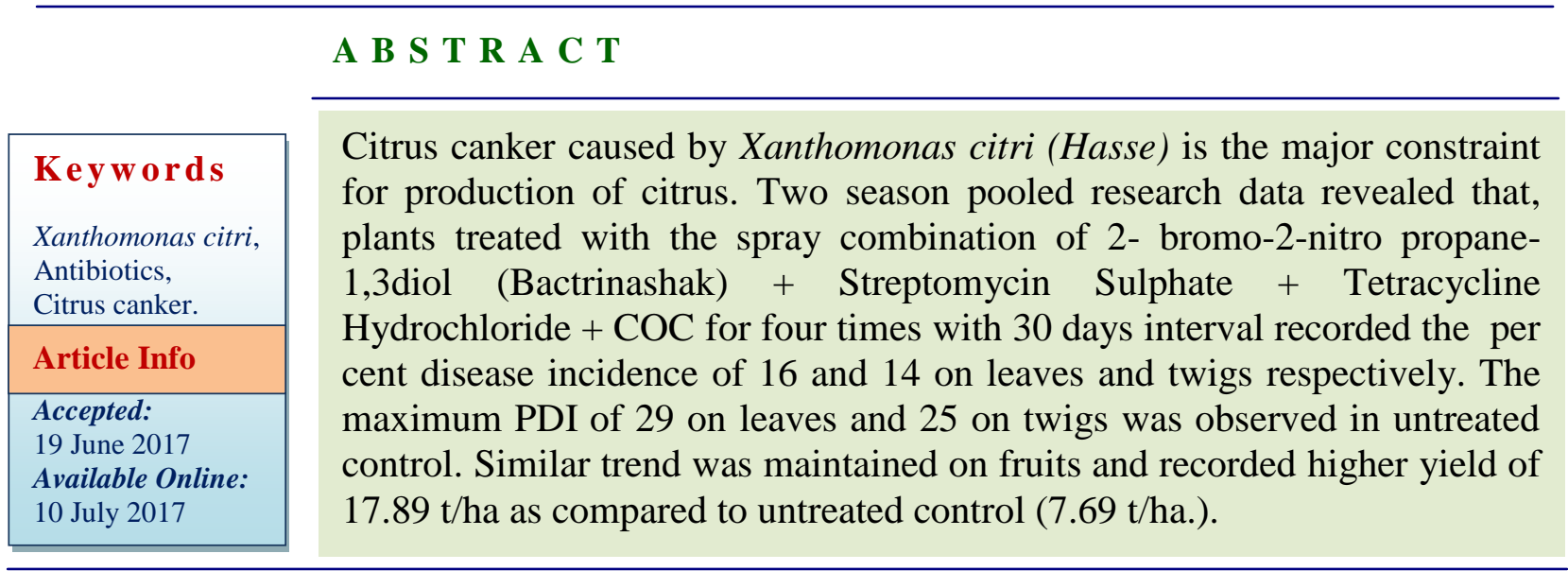

\section{Introduction}

Citrus is an important crop in Karnataka, where it is produced for both domestic consumption and regional markets. It was grown since ancient times for its nutrition and medicinal value. In India the total area under citrus is about 10.42 lakh hectares and producing 100 lakh tonnes with the productivity of 9.68 tonnes per hectare. Citrus is cultivated practically in every state. However, Maharastra, Andrapradesh, Karnataka, Punjab and Assam are leading citrus growing states. Karnataka stands $7^{\text {th }}$ major state in acreage with an area of 18.10 thousand hectare with the production of 399.0 thousand tonnes and productivity of 22.04 tonnes per hectare, citrus being important crop still there is an opportunity to increase the area under cultivation. Major diseases constraining the production of citrus are citrus canker, citrus greening, citrus tristeza and citrus nematode disease. Among them citrus canker, caused by Xanthomonas citri is one of the lethal disease in affecting all type of citrus crops (Prakash and Karemgam, 2012).

Canker-affected citrus specimens collected from Dehra Dun during 1827 to 1831 and from Java during 1842 to 1844 (Fawcett and Jenkins, 1933) suggests the origin of canker either in India or Java or in some other parts of Asia. However, canker disease also noticed in Northern Uganda in 2010 (Anonymous, 2012) where it was first detected in an orchard in lira district. The 
disease however has spread to all citrus growing areas in the region.

In India, Luthra and Sattar (1942) and Bedi (1961) from Punjab, Ramakrishnan (1954) from Tamil Nadu, Govinda Rao (1954) from Andhra Pradesh, Venkatakrishnaiah (1957) and Aiyappa (1958) from Karnataka, Prasad (1959) from Rajasthan, Parsai (1959) from Madhya Pradesh, Chowdhury (1951) from Assam, Nirvan (1960) from Uttar Pradesh and several others have reported the incidence of canker on the acid lime and several other varieties of citrus. Further, canker is reported to appear as a serious problem wherever citrus is grown on a large scale.

Citrus canker is a characterized by the occurrence of conspicuously raised necrotic lesions on leaves, twigs and fruits (Schubert, et.al., 2000) Nikhil et.al., 2013, reported Corky pustules developed on leaves, twigs, thorns and fruits were turn light tan to brown corky cankers, which were rough to touch. Often a water socked margins developing around the Necrotic tissue.

In recent years, due to severe and epidemic outbreak of the disease many farmers have uprooted the plants and destroying the orchards. The disease causes extensive damage to citrus and severity of disease varies with species, varieties and prevailing climate conditions. Thus there is an urgent need to developed suitable location specific management practices to mitigate the problem. Hence, the present work was carried out to identify the suitable management schedule in context to citrus canker.

\section{Materials and Methods}

The experiment site/orchard located at Jajurayanhalli, Pavagada, Tumkur, Karnataka which was one $\mathrm{km}$ away from Agricultural Research Station, Pavagada. The trials were carried out in two sites in the orchard for two years where 80-100 per cent trees were infested with citrus canker at the beginning of the experiment.

Field trials were conducted during Rabi season of $2014 \& 2015$. The treatments were framed and imposed with different concentrations. In each treatment, ten plants were maintained which constitute the replications. Plants were sprayed with different combinations of antibiotics at 30 days interval and observation were recorded on disease incidence and severity on leaves, twigs and fruits before the initiation of treatment and also at regular intervals of 30 days. The per cent disease incidence was calculated as per the formulae given by Wheeler, 1969

\section{Results and Discussion}

Experimental results of two season pooled research data (Table 1a and $1 \mathrm{~b}$ ) revealed that, before the initiation of the treatments the per cent disease incidence (PDI) on leaves and twigs were in the range of 22 to 25 and 17 to 20 , respectively. At the end of fourth spray Least PDI was observed in plants treated with the spray combination of 2-bromo- 2-nitro propane-1,3 diol (Bactrinashak) + Streptomycin Sulphate + Tetracycline Hydrochloride $+\mathrm{COC}\left(\mathrm{T}_{9}\right)$ followed by plants sprayed with Bactoson + Streptomycin Sulphate + Tetracycline Hydrochloride + COC $\left(\mathrm{T}_{8}\right)$ with values of 16 and 19 per cent respectively on leaves and 14 percent in both the treatments on twigs. The maximum PDI of 29 on leaves and 25 on twigs was observed in untreated control $\left(\mathrm{T}_{10}\right)$. 
Table.1a Pooled data on the evaluation of different antibiotics and bactericides and combinations for the management of citrus canker

a. Percent disease incidence on leaves

\begin{tabular}{|c|c|c|c|c|c|c|c|c|}
\hline \multirow[t]{2}{*}{ Sl.No } & \multirow[t]{2}{*}{ Treatments } & \multirow[t]{2}{*}{ Dosage } & \multicolumn{5}{|c|}{$\begin{array}{l}\text { Per cent disease incidence on leaves after } 1^{\text {st }} \text { to } \\
4^{\text {th }} \text { spray }\end{array}$} & \multirow{2}{*}{$\begin{array}{l}\text { Percent } \\
\text { disease } \\
\text { reduction }\end{array}$} \\
\hline & & & $\begin{array}{l}\text { Before } \\
\text { sprav }\end{array}$ & $\begin{array}{c}\text { Ist } \\
\text { sprav }\end{array}$ & $\begin{array}{c}2^{\text {nd }} \\
\text { sprav }\end{array}$ & $\begin{array}{c}3^{\text {rd }} \\
\text { sprav }\end{array}$ & $\begin{array}{l}4^{\text {th }} \\
\text { sprav }\end{array}$ & \\
\hline 1 & $\begin{array}{l}\text { Sreptocycline Sulphate }+ \text { Tetracycline } \\
\text { Hydrochloride }\end{array}$ & $3 \mathrm{~g} / \mathrm{L}$ & $\begin{array}{c}25 \\
(5.05)^{\mathrm{c}}\end{array}$ & $\begin{array}{c}21 \\
(4.64)^{b}\end{array}$ & $\begin{array}{c}20 \\
(4.53)^{\mathrm{ab}}\end{array}$ & $\begin{array}{c}20.5 \\
(4.58)^{\mathrm{bc}}\end{array}$ & $\begin{array}{c}20 \\
(4.53)^{\mathrm{c}}\end{array}$ & 56.9 \\
\hline 2 & $\begin{array}{l}\text { 2- bromo-2-nitro propane- } \\
\text { 1,3diol(Bactrinashak) }\end{array}$ & $2 \mathrm{~g} / \mathrm{L}$ & $\begin{array}{c}25 \\
(5.05)^{\mathrm{c}}\end{array}$ & $\begin{array}{c}22 \\
(4.74)^{b}\end{array}$ & $\begin{array}{c}25 \\
(5.05)^{\mathrm{c}}\end{array}$ & $\begin{array}{c}23.5 \\
(4.90)^{\mathrm{ef}}\end{array}$ & $\begin{array}{c}21 \\
(4.64)^{\text {bef }}\end{array}$ & 44.4 \\
\hline 3 & Bactosan (Herbal Product) & $2 \mathrm{~g} / \mathrm{L}+3 \mathrm{~g} / \mathrm{L}$ & $\begin{array}{c}24.5 \\
(5.00)^{\mathrm{bc}}\end{array}$ & $\begin{array}{c}24 \\
(4.95)^{\mathrm{b}}\end{array}$ & $\begin{array}{c}25 \\
(5.05)^{\mathrm{c}}\end{array}$ & $\begin{array}{c}24.5 \\
(5.00)^{\mathrm{f}}\end{array}$ & $\begin{array}{c}22 \\
(4.74)^{\mathrm{f}}\end{array}$ & 40.2 \\
\hline 4 & Copper Oxy Chloride (COC) & $0.5 \mathrm{~g} / \mathrm{L}$ & $\begin{array}{c}23.5 \\
(4.90)^{\mathrm{abc}}\end{array}$ & $\begin{array}{c}23 \\
(4.85)^{b}\end{array}$ & $\begin{array}{c}22 \\
(4.74)^{\mathrm{c}}\end{array}$ & $\begin{array}{c}22.5 \\
(4.80)^{\mathrm{ef}}\end{array}$ & $\begin{array}{c}23 \\
(4.85)^{\mathrm{f}}\end{array}$ & 40.2 \\
\hline 5 & $\begin{array}{l}\text { Sreptocycline Sulphate }+ \text { Tetracycline } \\
\text { Hydrochloride }+ \text { COC }\end{array}$ & $\begin{array}{l}0.5 \mathrm{~g} / \mathrm{L}+3 \mathrm{~g} / \\
\mathrm{L}\end{array}$ & $\begin{array}{c}22.5 \\
(4.80)^{\mathrm{ab}}\end{array}$ & $\begin{array}{c}20 \\
(4.53)^{\mathrm{a}}\end{array}$ & $\begin{array}{c}20 \\
(4.53)^{\mathrm{a}}\end{array}$ & $\begin{array}{c}20 \\
(4.53)^{\mathrm{b}}\end{array}$ & $\begin{array}{c}19 \\
(4.42)^{\mathrm{b}}\end{array}$ & 61.1 \\
\hline 6 & Bactrinashak+ COC & $0.5 \mathrm{~g} / \mathrm{L}$ & $\begin{array}{c}22 \\
(4.74)^{\mathrm{abc}}\end{array}$ & $\begin{array}{c}21 \\
(4.64)^{\mathrm{a}}\end{array}$ & $\begin{array}{c}21 \\
(4.64) \mathrm{bc}\end{array}$ & $\begin{array}{c}21 \\
(4.64)^{\mathrm{cd}}\end{array}$ & $\begin{array}{c}21 \\
(4.64)^{\mathrm{cd}}\end{array}$ & 51.3 \\
\hline 7 & Bactosan+ COC & $\begin{array}{l}0.5 \mathrm{~g} / \mathrm{L}+ \\
3 \mathrm{~g} / \mathrm{L}\end{array}$ & $\begin{array}{c}24 \\
(4.95)^{\mathrm{abc}}\end{array}$ & $\begin{array}{c}21 \\
(4.64)^{\mathrm{ab}}\end{array}$ & $\begin{array}{c}23 \\
(4.85)^{\mathrm{c}}\end{array}$ & $\begin{array}{c}22 \\
(4.74) \text { de }\end{array}$ & $\begin{array}{c}21 \\
(4.64)^{\mathrm{de}}\end{array}$ & 47.2 \\
\hline 8 & $\begin{array}{l}\text { Bactosan + Sreptocycline Sulphate + } \\
\text { Tetracycline Hydrochloride + COC }\end{array}$ & $\begin{array}{l}0.5 \mathrm{~g} / \mathrm{L}+0.5 \\
\mathrm{~g} / \mathrm{L}+3 \mathrm{~g} / \mathrm{L}\end{array}$ & $\begin{array}{c}24 \\
(4.95)^{\mathrm{abc}}\end{array}$ & $\begin{array}{c}21 \\
(4.64)^{b}\end{array}$ & $\begin{array}{c}20 \\
(4.53)^{\mathrm{ab}}\end{array}$ & $\begin{array}{c}20.5 \\
(4.58)^{\mathrm{bc}}\end{array}$ & $\begin{array}{c}19 \\
(4.42)^{b}\end{array}$ & 58.3 \\
\hline 9 & $\begin{array}{l}\text { Bactrinashak+ Sreptocycline } \\
\text { Sulphate + Tetracycline } \\
\text { Hydrochloride + COC }\end{array}$ & $\begin{array}{l}2.0 \mathrm{~g} / \mathrm{L}+0.5 \\
\mathrm{~g} / \mathrm{L}+3 \mathrm{~g} / \mathrm{L}\end{array}$ & $\begin{array}{c}25 \\
(5.05)^{\mathrm{c}}\end{array}$ & $\begin{array}{c}20 \\
(4.53)^{\mathrm{b}}\end{array}$ & $\begin{array}{c}21 \\
(4.64)^{\mathrm{a}}\end{array}$ & $\begin{array}{c}20.5 \\
(4.58)^{\mathrm{a}}\end{array}$ & $\begin{array}{c}16 \\
(4.06)^{\mathrm{a}}\end{array}$ & 70.8 \\
\hline 10 & Control & - & $\begin{array}{c}25.5 \\
(5.10)^{\mathrm{c}}\end{array}$ & $\begin{array}{c}26 \\
(5.15)^{\mathrm{c}}\end{array}$ & $\begin{array}{c}29 \\
(5.43)^{\mathrm{d}}\end{array}$ & $\begin{array}{c}27.5 \\
(5.29)^{\mathrm{g}}\end{array}$ & $\begin{array}{c}29 \\
(5.43)^{\mathrm{g}}\end{array}$ & - \\
\hline & SEM \pm & & 1.06 & 0.97 & 0.99 & 0.98 & 1.00 & \\
\hline & CD 0.05 & & 2.15 & 1.97 & 2.02 & 1.99 & 2.04 & \\
\hline & CD 0.01 & & 2.88 & 2.64 & 2.70 & 2.66 & 3.86 & \\
\hline & $\mathbf{C V}$ & & 6.87 & 7.31 & 8.20 & 8.26 & 9.41 & \\
\hline
\end{tabular}


Table.1b Pooled data on the evaluation of different antibiotics and bactericides and combinations for the management of citrus canker

b. Percent disease incidence on twigs

\begin{tabular}{|c|c|c|c|c|c|c|c|c|}
\hline \multirow[t]{2}{*}{ Sl.No } & \multirow[t]{2}{*}{ Treatments } & \multirow[t]{2}{*}{ Dosage } & \multicolumn{5}{|c|}{$\begin{array}{l}\text { Per cent disease incidence on twigs after } 1^{\text {st }} \text { to } \\
\qquad 4^{\text {th }} \text { spray }\end{array}$} & \multirow{2}{*}{$\begin{array}{l}\text { Percent } \\
\text { disease } \\
\text { reduction }\end{array}$} \\
\hline & & & $\begin{array}{l}\text { Before } \\
\text { spray }\end{array}$ & $\begin{array}{c}\text { Ist } \\
\text { spray }\end{array}$ & $\begin{array}{c}2^{\text {nd }} \\
\text { spray }\end{array}$ & $\begin{array}{c}3^{\text {rd }} \\
\text { spray }\end{array}$ & $\begin{array}{l}4^{\text {th }} \\
\text { spray }\end{array}$ & \\
\hline 1 & $\begin{array}{l}\text { Sreptocycline Sulphate + Tetracycline } \\
\text { Hydrochloride }\end{array}$ & $3 g / L$ & $\begin{array}{c}20.5 \\
(4.58)\end{array}$ & $\begin{array}{c}17 \\
(4.18)^{\mathrm{abc}}\end{array}$ & $\begin{array}{c}17 \\
(4.18)^{\mathrm{b}}\end{array}$ & $\begin{array}{c}17 \\
(4.18)^{\mathrm{b}}\end{array}$ & $\begin{array}{c}15 \\
(3.94)^{\mathrm{cd}}\end{array}$ & 51.8 \\
\hline 2 & $\begin{array}{l}\text { 2- bromo-2-nitro propane- } \\
\text { 1,3diol(Bactrinashak) }\end{array}$ & $2 \mathrm{~g} / \mathrm{L}$ & $\begin{array}{c}18 \\
(4.30)\end{array}$ & $\begin{array}{c}17 \\
(4.18)^{\mathrm{abc}}\end{array}$ & $\begin{array}{c}18 \\
(4.30)^{\mathrm{c}}\end{array}$ & $\begin{array}{c}17.5 \\
(4.24)^{\mathrm{bc}}\end{array}$ & $\begin{array}{c}17 \\
(4.18)^{\mathrm{e}}\end{array}$ & 42.5 \\
\hline 3 & Bactosan (Herbal Product) & $2 g / L+3 g / L$ & $\begin{array}{c}17 \\
(4.18)\end{array}$ & $\begin{array}{c}20 \\
(4.53)^{\mathrm{c}} \\
\end{array}$ & $\begin{array}{c}17 \\
(4.18)^{\mathrm{c}}\end{array}$ & $\begin{array}{c}18.5 \\
(4.36)^{\mathrm{bc}}\end{array}$ & $\begin{array}{c}17 \\
(4.18)^{\mathrm{e}} \\
\end{array}$ & 38.8 \\
\hline 4 & Copper Oxy Chloride (COC) & $0.5 \mathrm{~g} / \mathrm{L}$ & $\begin{array}{c}18.5 \\
(4.36)\end{array}$ & $\begin{array}{c}18 \\
(4.30)^{\mathrm{bc}}\end{array}$ & $\begin{array}{c}18 \\
(4.30)^{\mathrm{c}}\end{array}$ & $\begin{array}{c}18 \\
(4.12)^{\mathrm{c}}\end{array}$ & $\begin{array}{c}17 \\
(4.18)^{\mathrm{e}}\end{array}$ & 38.8 \\
\hline 5 & $\begin{array}{l}\text { Sreptocycline Sulphate + Tetracycline } \\
\text { Hydrochloride + COC }\end{array}$ & $\begin{array}{l}0.5 \mathrm{~g} / \mathrm{L}+3 \mathrm{~g} / \\
\mathrm{L}\end{array}$ & $\begin{array}{c}18 \\
(4.30)\end{array}$ & $\begin{array}{c}14 \\
(3.81)^{\mathrm{ab}}\end{array}$ & $\begin{array}{c}19 \\
(4.42)^{\mathrm{b}}\end{array}$ & $\begin{array}{c}16.5 \\
(4.12)^{\mathrm{b}}\end{array}$ & $\begin{array}{c}13 \\
(3.67)^{\mathrm{bc}}\end{array}$ & 57.4 \\
\hline 6 & Bactrinashak+ COC & $0.5 \mathrm{~g} / \mathrm{L}$ & $\begin{array}{c}17.5 \\
(4.24)\end{array}$ & $\begin{array}{c}15 \\
(3.94)^{\mathrm{ab}}\end{array}$ & $\begin{array}{c}17 \\
(4.18)^{\mathrm{bc}}\end{array}$ & $\begin{array}{c}16 \\
(4.06)^{\mathrm{bc}}\end{array}$ & $\begin{array}{c}15 \\
(3.94)^{\mathrm{de}}\end{array}$ & 46.2 \\
\hline 7 & Bactosan+ COC & $\begin{array}{l}0.5 \mathrm{~g} / \mathrm{L}+ \\
3 \mathrm{~g} / \mathrm{L}\end{array}$ & $\begin{array}{c}19 \\
(4.42)\end{array}$ & $\begin{array}{c}18 \\
(4.30)^{\mathrm{c}}\end{array}$ & $\begin{array}{c}18 \\
(4.30)^{\mathrm{c}}\end{array}$ & $\begin{array}{c}18 \\
(4.30)^{\mathrm{c}}\end{array}$ & $\begin{array}{c}17 \\
(4.17)^{\mathrm{de}}\end{array}$ & 44.4 \\
\hline 8 & $\begin{array}{l}\text { Bactosan + Sreptocycline Sulphate + } \\
\text { Tetracycline Hydrochloride + COC }\end{array}$ & $\begin{array}{l}0.5 \mathrm{~g} / \mathrm{L}+0.5 \\
\mathrm{~g} / \mathrm{L}+3 \mathrm{~g} / \mathrm{L}\end{array}$ & $\begin{array}{c}17.5 \\
(4.24)\end{array}$ & $\begin{array}{c}18 \\
(4.30)^{\mathrm{a}}\end{array}$ & $\begin{array}{c}14 \\
(3.81)^{\mathrm{a}}\end{array}$ & $\begin{array}{c}16 \\
(4.06)^{\mathrm{a}}\end{array}$ & $\begin{array}{c}14 \\
(3.81)^{\mathrm{ab}}\end{array}$ & 62.9 \\
\hline 9 & $\begin{array}{l}\text { Bactrinashak+ Sreptocycline } \\
\text { Sulphate + Tetracycline } \\
\text { Hydrochloride + COC }\end{array}$ & $\begin{array}{l}2.0 \mathrm{~g} / \mathrm{L}+0.5 \\
\mathrm{~g} / \mathrm{L}+3 \mathrm{~g} / \mathrm{L}\end{array}$ & $\begin{array}{c}18 \\
(4.30)\end{array}$ & $\begin{array}{c}17 \\
(4.18)^{\mathrm{a}}\end{array}$ & $\begin{array}{c}15 \\
(3.94)^{\mathrm{a}}\end{array}$ & $\begin{array}{c}16 \\
(4.06)^{\mathrm{a}}\end{array}$ & $\begin{array}{c}14 \\
(3.81)^{\mathrm{a}}\end{array}$ & 68.5 \\
\hline 10 & Control & - & $\begin{array}{c}19.5 \\
(4.47)\end{array}$ & $\begin{array}{c}23 \\
(4.85)^{\mathrm{d}}\end{array}$ & $\begin{array}{c}21 \\
(4.64)^{d}\end{array}$ & $\begin{array}{c}22 \\
(4.74)^{\mathrm{d}}\end{array}$ & $\begin{array}{c}25 \\
(5.05)^{\mathrm{f}}\end{array}$ & - \\
\hline & SEM \pm & & 1.13 & 0.90 & 0.97 & 0.70 & 1.03 & \\
\hline & CD 0.05 & & 2.29 & $\mathbf{1 . 8 3}$ & 1.96 & 1.43 & 2.08 & \\
\hline & CD 0.01 & & 3.06 & 2.46 & 2.63 & 1.91 & 2.79 & \\
\hline & $\mathrm{CV}$ & & 10.85 & 8.59 & 10.39 & 15.64 & 11.99 & \\
\hline
\end{tabular}


Table.1c Pooled data on the evaluation of different antibiotics and bactericides and combinations for the management of citrus canker

c. Percent disease incidence on fruits

\begin{tabular}{|c|c|c|c|c|c|c|c|c|}
\hline \multirow[t]{2}{*}{$\begin{array}{l}\text { Sl. } \\
\text { No }\end{array}$} & \multirow[t]{2}{*}{ Treatments } & \multirow[t]{2}{*}{ Dosage } & \multicolumn{5}{|c|}{$\begin{array}{l}\text { Per cent disease incidence on fruits after } 1^{\text {st }} \text { to } \\
\qquad 4^{\text {th }} \text { spray }\end{array}$} & \multirow{2}{*}{$\begin{array}{l}\text { Percent } \\
\text { disease } \\
\text { reduction }\end{array}$} \\
\hline & & & $\begin{array}{l}\text { Before } \\
\text { spray }\end{array}$ & $\begin{array}{c}\text { Ist } \\
\text { spray }\end{array}$ & $\begin{array}{c}2^{\text {nd }} \\
\text { spray }\end{array}$ & $\begin{array}{c}3^{\text {rd }} \\
\text { spray }\end{array}$ & $\begin{array}{l}4^{\text {th }} \\
\text { spray }\end{array}$ & \\
\hline 1 & $\begin{array}{l}\text { Sreptocycline Sulphate + Tetracycline } \\
\text { Hydrochloride }\end{array}$ & $3 g / L$ & $\begin{array}{c}21.5 \\
(4.69)^{\mathrm{bc}}\end{array}$ & - & - & $\begin{array}{c}12.5 \\
(3.61)^{\mathrm{b}}\end{array}$ & $\begin{array}{c}11 \\
(3.39)^{\mathrm{cd}}\end{array}$ & 62.9 \\
\hline 2 & $\begin{array}{l}\text { 2- bromo-2-nitro propane- } \\
\text { 1,3diol(Bactrinashak) }\end{array}$ & $2 \mathrm{~g} / \mathrm{L}$ & $\begin{array}{c}24 \\
(4.95)^{\mathrm{d}}\end{array}$ & - & - & $\begin{array}{c}12.5 \\
(3.61)^{\mathrm{b}}\end{array}$ & $\begin{array}{c}13 \\
(3.67)^{\mathrm{ef}}\end{array}$ & $\mathbf{5 0}$ \\
\hline 3 & Bactosan (Herbal Product) & $2 \mathrm{~g} / \mathrm{L}+3 \mathrm{~g} / \mathrm{L}$ & $\begin{array}{c}19 \\
(4.42)^{\mathrm{a}}\end{array}$ & - & - & $\begin{array}{c}13 \\
(3.67)^{\mathrm{b}}\end{array}$ & $\begin{array}{c}14 \\
(3.81)^{\mathrm{fg}}\end{array}$ & 46.4 \\
\hline 4 & Copper Oxy Chloride (COC) & $0.5 \mathrm{~g} / \mathrm{L}$ & $\begin{array}{c}20.5 \\
(4.58)^{\mathrm{ab}}\end{array}$ & - & - & $\begin{array}{c}13.5 \\
(3.74)^{\mathrm{b}}\end{array}$ & $\begin{array}{c}18 \\
(3.40)^{\mathrm{g}}\end{array}$ & 39.2 \\
\hline 5 & $\begin{array}{l}\text { Sreptocycline Sulphate + Tetracycline } \\
\text { Hydrochloride + COC }\end{array}$ & $\begin{array}{l}0.5 \mathrm{~g} / \mathrm{L}+3 \mathrm{~g} / \\
\mathrm{L}\end{array}$ & $\begin{array}{c}22.5 \\
(4.80)^{\mathrm{bcd}}\end{array}$ & - & - & $\begin{array}{c}13 \\
(3.67)^{\mathrm{b}}\end{array}$ & $\begin{array}{c}10 \\
(3.24)^{\mathrm{bc}}\end{array}$ & 66 \\
\hline 6 & Bactrinashak+ COC & $0.5 \mathrm{~g} / \mathrm{L}$ & $\begin{array}{c}22 \\
(4.74)^{\text {bcd }}\end{array}$ & - & - & $\begin{array}{c}14.5 \\
(3.87)^{\mathrm{b}}\end{array}$ & $\begin{array}{c}13 \\
(3.67)^{\mathrm{de}}\end{array}$ & 55.3 \\
\hline 7 & Bactosan+ COC & $\begin{array}{l}0.5 \mathrm{~g} / \mathrm{L}+ \\
3 \mathrm{~g} / \mathrm{L}\end{array}$ & $\begin{array}{c}22 \\
(4.74)^{\mathrm{bcd}}\end{array}$ & - & - & $\begin{array}{c}13.5 \\
(3.74)^{\mathrm{b}}\end{array}$ & $\begin{array}{c}14 \\
(3.81)^{\mathrm{ef}}\end{array}$ & 48.2 \\
\hline 8 & $\begin{array}{l}\text { Bactosan + Sreptocycline Sulphate + } \\
\text { Tetracycline Hydrochloride + COC }\end{array}$ & $\begin{array}{l}0.5 \mathrm{~g} / \mathrm{L}+0.5 \\
\mathrm{~g} / \mathrm{L}+3 \mathrm{~g} / \mathrm{L}\end{array}$ & $\begin{array}{c}21 \\
(4.64)^{\mathrm{ab}} \\
\end{array}$ & - & - & $\begin{array}{c}10 \\
(3.24)^{\mathrm{a}} \\
\end{array}$ & $\begin{array}{c}9 \\
(3.08)^{\mathrm{b}} \\
\end{array}$ & 71.4 \\
\hline 9 & $\begin{array}{l}\text { Bactrinashak+ Sreptocycline } \\
\text { Sulphate + Tetracycline } \\
\text { Hydrochloride + COC }\end{array}$ & $\begin{array}{l}2.0 \mathrm{~g} / \mathrm{L}+0.5 \\
\mathrm{~g} / \mathrm{L}+3 \mathrm{~g} / \mathrm{L}\end{array}$ & $\begin{array}{c}21 \\
(4.64)^{a b}\end{array}$ & - & - & $\begin{array}{c}9 \\
(3.08)^{\mathrm{a}}\end{array}$ & $\begin{array}{c}6 \\
\left(2.55^{\mathrm{a}}\right. \\
\end{array}$ & 80.3 \\
\hline 10 & Control & - & $\begin{array}{c}23.5 \\
(4.90)^{\mathrm{cd}} \\
\end{array}$ & - & - & $\begin{array}{c}25.5 \\
(5.10)^{\mathrm{c}} \\
\end{array}$ & $\begin{array}{c}27 \\
(5.24)^{\mathrm{h}}\end{array}$ & - \\
\hline & SEM \pm & & 1.07 & & & 1.07 & 1.01 & \\
\hline & CD 0.05 & & 2.17 & & & 2.18 & 2.05 & \\
\hline & CD 0.01 & & 2.91 & & & 2.92 & 2.75 & \\
\hline & CV & & 8.68 & & & 13.98 & 12.62 & \\
\hline
\end{tabular}


Table.2 Evaluation of different antibiotics and bactericides and combinations for the management of citrus canker for the year 2014 and 15

\begin{tabular}{|c|c|c|c|c|c|c|c|c|}
\hline Sl.No & Treatments & Dosage & \multicolumn{6}{|c|}{ Percent disease reduction } \\
\hline & & & \multicolumn{2}{|c|}{ On leaves } & \multicolumn{2}{|c|}{ On twig } & \multicolumn{2}{|c|}{ On fruit } \\
\hline & & & 2014 & 2015 & 2014 & 2015 & 2014 & 2015 \\
\hline 1 & $\begin{array}{l}\text { Sreptocycline Sulphate + Tetracycline } \\
\text { Hydrochloride }\end{array}$ & $3 g / L$ & 51.42 & 62.16 & $\mathbf{5 3 . 5 7}$ & 50.00 & 59.25 & 62.06 \\
\hline 2 & $\begin{array}{l}\text { 2- bromo-2-nitro propane- } \\
\text { 1,3diol(Bactrinashak) }\end{array}$ & $2 \mathrm{~g} / \mathrm{L}$ & 42.85 & 45.94 & 46.42 & 38.46 & 51.85 & 48.27 \\
\hline 3 & Bactosan (Herbal Product) & $2 \mathrm{~g} / \mathrm{L}+3 \mathrm{~g} / \mathrm{L}$ & 40.00 & 40.54 & 39.28 & 38.46 & 48.14 & 44.82 \\
\hline 4 & Copper Oxy Chloride (COC) & $0.5 \mathrm{~g} / \mathrm{L}$ & 40.00 & 43.24 & 42.85 & 34.61 & 33.33 & 44.82 \\
\hline 5 & $\begin{array}{l}\text { Sreptocycline Sulphate + Tetracycline } \\
\text { Hydrochloride + COC }\end{array}$ & $\begin{array}{l}0.5 \mathrm{~g} / \mathrm{L}+3 \mathrm{~g} / \\
\mathrm{L}\end{array}$ & 54.28 & 67.56 & 57.00 & 57.69 & 62.96 & 68.96 \\
\hline 6 & Bactrinashak+ COC & $0.5 \mathrm{~g} / \mathrm{L}$ & 45.70 & 56.75 & 50.00 & 42.30 & 51.85 & 58.62 \\
\hline 7 & Bactosan+ COC & $\begin{array}{l}0.5 \mathrm{~g} / \mathrm{L}+ \\
3 \mathrm{~g} / \mathrm{L}\end{array}$ & 45.71 & 48.64 & 46.42 & 42.30 & 48.14 & 48.27 \\
\hline 8 & $\begin{array}{l}\text { Bactosan + Sreptocycline Sulphate + } \\
\text { Tetracycline Hydrochloride + COC }\end{array}$ & $\begin{array}{l}0.5 \mathrm{~g} / \mathrm{L}+0.5 \\
\mathrm{~g} / \mathrm{L}+3 \mathrm{~g} / \mathrm{L}\end{array}$ & 60.00 & 56.75 & 60.71 & 65.38 & 66.66 & 75.86 \\
\hline 9 & $\begin{array}{l}\text { Bactrinashak+ Sreptocycline } \\
\text { Sulphate + Tetracycline } \\
\text { Hydrochloride + COC }\end{array}$ & $\begin{array}{l}2.0 \mathrm{~g} / \mathrm{L}+0.5 \\
\mathrm{~g} / \mathrm{L}+3 \mathrm{~g} / \mathrm{L}\end{array}$ & 68.57 & 72.97 & 67.85 & 69.23 & 77.77 & 82.75 \\
\hline 10 & Control & - & - & - & - & - & - & - \\
\hline
\end{tabular}


Table.3 Pooled data on the evaluation of different antibiotics and bactericides and combinations for the management of citrus canker

\section{Pooled Yield data}

\begin{tabular}{|c|c|c|c|c|c|}
\hline $\begin{array}{l}\text { Sl. } \\
\text { No }\end{array}$ & Treatments & Dosage & $\begin{array}{l}\text { Total fruit } \\
\text { yield per } \\
\text { plant }\end{array}$ & $\begin{array}{l}\text { Disease free } \\
\text { fruit yield per } \\
\text { plant }(\mathrm{kg})\end{array}$ & $\begin{array}{l}\text { Fruit yield } \\
\quad(\mathrm{t} / \mathrm{ha})\end{array}$ \\
\hline 1 & $\begin{array}{l}\text { Sreptocycline Sulphate }+ \text { Tetracycline } \\
\text { Hydrochloride }\end{array}$ & $3 g / L$ & $\begin{array}{c}41.1 \\
(6.45)^{\mathrm{e}}\end{array}$ & $\begin{array}{c}29.5 \\
(5.48)^{\text {cdef }}\end{array}$ & $\begin{array}{l}12.53 \\
(3.61)\end{array}$ \\
\hline 2 & $\begin{array}{l}\text { 2- bromo-2-nitro propane- } \\
\text { 1,3diol(Bactrinashak) }\end{array}$ & $2 \mathrm{~g} / \mathrm{L}$ & $\begin{array}{c}39.5 \\
(6.32)^{\mathrm{f}}\end{array}$ & $\begin{array}{c}27 \\
(5.24)^{\mathrm{def}}\end{array}$ & $\begin{array}{l}11.47 \\
(3.46)\end{array}$ \\
\hline 3 & Bactosan (Herbal Product) & $2 \mathrm{~g} / \mathrm{L}+3 \mathrm{~g} / \mathrm{L}$ & $\begin{array}{c}37 \\
(6.12)^{\mathrm{g}}\end{array}$ & $\begin{array}{c}25.1 \\
(5.06)^{f}\end{array}$ & $\begin{array}{l}10.66 \\
(3.34)\end{array}$ \\
\hline 4 & Copper Oxy Chloride (COC) & $0.5 \mathrm{~g} / \mathrm{L}$ & $\begin{array}{c}38.7 \\
(6.26)^{\mathrm{f}}\end{array}$ & $\begin{array}{c}26.9 \\
(5.23)^{\mathrm{ef}}\end{array}$ & $\begin{array}{l}11.43 \\
(3.45)\end{array}$ \\
\hline 5 & $\begin{array}{l}\text { Sreptocycline Sulphate + Tetracycline } \\
\text { Hydrochloride + COC }\end{array}$ & $\begin{array}{l}0.5 \mathrm{~g} / \mathrm{L}+3 \mathrm{~g} / \\
\mathrm{L}\end{array}$ & $\begin{array}{c}45 \\
(6.75)^{\mathrm{c}}\end{array}$ & $\begin{array}{c}33 \\
(5.79)^{\text {bcd }}\end{array}$ & $\begin{array}{r}14.02 \\
(3.81)\end{array}$ \\
\hline 6 & Bactrinashak+ COC & $0.5 \mathrm{~g} / \mathrm{L}$ & $\begin{array}{c}45.5 \\
(6.78)^{\mathrm{c}}\end{array}$ & $\begin{array}{c}35.6 \\
(6.01)^{\mathrm{b}}\end{array}$ & $\begin{array}{l}15.13 \\
(3.95)\end{array}$ \\
\hline 7 & Bactosan+ COC & $\begin{array}{l}0.5 \mathrm{~g} / \mathrm{L}+ \\
3 \mathrm{~g} / \mathrm{L}\end{array}$ & $\begin{array}{c}42.6 \\
(6.57)^{\mathrm{d}}\end{array}$ & $\begin{array}{c}31.6 \\
(5.67)^{\text {bcde }}\end{array}$ & $\begin{array}{l}13.43 \\
(3.73)\end{array}$ \\
\hline 8 & $\begin{array}{l}\text { Bactosan + Sreptocycline Sulphate + } \\
\text { Tetracycline Hydrochloride + COC }\end{array}$ & $\begin{array}{l}0.5 \mathrm{~g} / \mathrm{L}+0.5 \\
\mathrm{~g} / \mathrm{L}+3 \mathrm{~g} / \mathrm{L}\end{array}$ & $\begin{array}{c}47.3 \\
(6.91)^{\mathrm{b}}\end{array}$ & $\begin{array}{l}34.6 \\
(5.92)^{\mathrm{bc}}\end{array}$ & $\begin{array}{c}14.7 \\
(3.90)\end{array}$ \\
\hline 9 & $\begin{array}{l}\text { Bactrinashak+ Sreptocycline } \\
\text { Sulphate + Tetracycline } \\
\text { Hydrochloride + COC }\end{array}$ & $\begin{array}{l}2.0 \mathrm{~g} / \mathrm{L}+0.5 \\
\mathrm{~g} / \mathrm{L}+3 \mathrm{~g} / \mathrm{L}\end{array}$ & $\begin{array}{l}53.25 \\
(7.33)^{\mathrm{a}} \\
\end{array}$ & $\begin{array}{c}42.1 \\
(6.53)^{\mathrm{a}}\end{array}$ & $\begin{array}{l}17.89 \\
(4.29) \\
\end{array}$ \\
\hline 10 & Control & - & $\begin{array}{c}30.1 \\
(5.53)^{\mathrm{h}}\end{array}$ & $\begin{array}{c}18.1 \\
(4.31)^{\mathrm{g}}\end{array}$ & $\begin{array}{c}7.69 \\
(2.86)\end{array}$ \\
\hline & SEM \pm & & 0.59 & 2.68 & 2.38 \\
\hline & CD 0.05 & & 1.34 & 6.07 & 5.39 \\
\hline & CD 0.01 & & 1.93 & 2.72 & 7.75 \\
\hline & $\mathbf{C V}$ & & 1.42 & 8.84 & 18.50 \\
\hline
\end{tabular}


The per cent disease reduction over control was maximum 70.80 and 68.50 per cent on leaves and twigs, respectively in $\mathrm{T}_{9}$ followed by $\mathrm{T}_{8}$ wherein we could record the PDI of 58.30 and 62.90 on leaves and twigs, respectively. Similar trend was also observed in both the seasons data (Table 2) wherein the per cent disease reduction over control was also maximum in $\mathrm{T}_{9}$ and $\mathrm{T}_{8}$ treated plants with 68.57 and 60.00, respectively on leaves whereas 67.85 and 60.71 on twigs during 2014. Similarly, during 2015 per cent disease reduction in $\mathrm{T}_{9}$ treated plants was 72.97 and 69.23 on leaves and twigs respectively. However, per cent disease incidence of 56.75 and 65.38 on leaves and twigs in plants treated with $\mathrm{T}_{8}$ treatment.

Per cent disease incidence on fruits: At the end of fourth spray, least PDI was recorded in $\mathrm{T}_{9}$ (6 per cent) followed by $\mathrm{T}_{8}$ ( 9 per cent) (Table 1c). However, maximum PDI on fruit was observed in untreated control i.e., 27 per cent. Similarly, the per cent disease reduction over control was maximum in $\mathrm{T}_{9}$ followed by $\mathrm{T}_{8}$ i.e., 80.30 and 71.40 per cent, respectively. Observation pertaining to fruit incidence for the year 2014 and 2015 were also shown that plants treated with the spray combination of 2- bromo-2-nitro propane-1,3diol $($ Bactrinashak $)+$ Streptomycin Sulphate + Tetracycline Hydrochloride + COC $\left(\mathrm{T}_{9}\right)$ was superior over untreated control followed by plants sprayed with Bactoson + Streptomycin Sulphate + Tetracycline Hydrochloride + COC $\left(\mathrm{T}_{8}\right)$ with the per cent disease reduction of 77.77 and 66.66, respectively for the year 2014 whereas 82.75 and 75.86 respectively for the year 2015 .

Plants treated with the spray combinations framed in Treatment $\mathrm{T}_{9}$ were recorded higher yield of $17.89 \mathrm{t} / \mathrm{ha}$ followed by plants treated with treatment $\mathrm{T}_{8}(14.7 \mathrm{t} / \mathrm{ha})$ as compared to untreated control where they could record only 7.69 t/ha (Table 3 ). The obtained results were in conformity with Leite et al., 1990 had reported that effective suppression of citrus canker by copper sprays. Rangaswani (1957) found streptomycin sulphate effective to check citrus canker at $1 \mathrm{mg}$ per $\mathrm{ml}$ in vitro and also reported 1 gram streptomycin or 2.5 gram of phytomycin per liter of water of were effective under field conditions. Patel and Padhya (1964) observed that three sprayings with a mixture of sodium arsenite and copper sulphate both at $100 \mathrm{ppm}$ during the season were effective in checking the spread of the disease. Streptomycin and streptochlor, Ziram were found to be effective against $X$. citri (Chakravarthi et al., as quoted by Rangaswami and Soumini Rajagopalan, 1973).

\section{Acknowledgements}

The service render by Mr. Ramachandra, Field Assistant, Mr Yusuf, Laboratory Assistant are greatly acknowledged. The help and support given by Mr. Panduragappa, owner of the experimental plantation is highly appreciated.

\section{References}

Aiyappa, K.M. 1958. Citrus cankerXanthomonas citri (Hasse) Dowson, Mysore Agric. J., 13: 164-67.

Anonymous. 2012. Ngetta ZARDI citrus pest and disease field indicator survey report. National Agricultural Research organization, Ministry of agriculture, Animal Industry and fisheries, Government of Uganda.

Bedi, K.S. 1961. Some important observations on the citrus canker in Punjab. Punjab Hort. J., 2: 89-91.

Chowdhury, S. 1951. Citrus canker in Assam. Pl. Prot. Bull., 3(3): 78-79.

Fawcett, H.S. and Jenkins, A.E. 1933. Records of citrus canker from herbarium specimens of the genus citrus 
in England and United States. Phytopathol., 23: 820-824.

Govinda Rao, P. 1954. Citrus diseases and their control in Andhra State. Andhra Agric. J., 1: 187-92.

Leite, Jr., R.P. 1990. Citrus canker: Prevention and control in the state of Parana. Fundacao IAPAR, Circular Instituto Agronomico do Parana. No. 61.

Luthra, J.C. and Sattar, A. 1942. Citrus canker and its control in Punjab. Punjab Fruit J., 6(1): 179-82.

Nikhil, P.N., Thomas, F.B., Jianwei, Q. and Mark, A. 2013. Estimation of citrus canker lesion size using hyperspectral reflectance imaging. Int. Agri. Engi. J., 22(3): 41-45.

Nirvan, R.S. 1960. Effect of antibiotic spray on citrus canker. Hort Adv., 4: 155-60.

Parsai, P.S. 1959. Citrus canker. Proceeding of the Seminar on Diseases of Horticultural Plants, Simla, pp. 91-95.

Patel, M.K. and Padhya, A.C. 1964. Sodim arsenite, copper sulphate spray for the control of citrus canker. Curr. Sci., 33: 87-8.

Prakash, M. and Karmegam, N. 2012. In-vitro antibacterial activity of certain plant extracts against plant disease causing bacteria isolated from citrus plant. Int. J. Curr. Microbiol. Appl. Sci., 1(1): 111.

Prasad. N. 1959. Citrus canker. Proceeding of the Seminar on Diseases of Horticultural Plants, Simla, pp. 86-8.

Ramakrishnan, T.S. 1954. Common Diseases of Citrus in Madras State. Publication of the Government of Madras.

Rangaswami, G. and Soumini Rajagopalan, C.K. 1973. Bacterial plant pathology, 291 pp. Tamil Nadu Agric. Univ., Coimbatore.

Rangaswami, G. 1957. Development of resistance to streptomycin in Xanthomonas citri and X. malvacearum. Curr. Sci., 26:185-6.

Schubert, T.S. and Miller, J.W 2000. Bacterial citrus canker. Gainesville, Florida, FDACS, Division of Plant Industry, USA.

Venkatakrishnaiah, N.S. 1957. Canker disease of sour lime and its control. J. Mysore Hort. Soc., 2(2, 3): 40-44.

Wheeler, B.E.J. 1969. An introduction to plant diseases John Wiley and Sons Limited, London, p. 301.

\section{How to cite this article:}

Jahir Basha, C.R. Prabhu, C. Ganiger and Soniya, M.C. 2017. Efficacy of Antibiotics and Bactericides for the Management of Xanthomonas citri (Hasse) Causing Citrus Canker. Int.J.Curr.Microbiol.App.Sci. 6(7): 1760-1768. doi: https://doi.org/10.20546/ijcmas.2017.607.212 